\title{
The effect of dexmedetomidine on GluR2 and NR2B expression of cortical neurons after cerebral ischemia-reperfusion in rats.
}

\author{
Yanwen $\mathrm{Li}^{1 *}$, Shikun Liu ${ }^{2}$ \\ ${ }^{1}$ Department of Anesthesiology, Third Xiangya Hospital of Central South University, Changsha, Hunan, PR China \\ ${ }^{2}$ Department of Pharmacy, the Third Xiangya Hospital of the Central South University, Changsha, Hunan, PR China
}

\begin{abstract}
Objective: Neurotransmitter plays a crucial role in ischemia cerebrovascular disease. Study has shown that Dexmedetomidine (Dex) contributes to neuroprotective functions. We thus observed the regulatory effect of Dex on the expressions of Glutamate Receptor-2 (GluR2) and N-Methyl-D-Aspartic Acid (NMDA) receptor 2B (NR2B) in cerebral cortical neurons with an ischemia-reperfusion rat model.

Patients and methods: Healthy male SD rats were randomly divided into sham, model and Dex treatment groups $(\mathrm{N}=20$ each). Whole-brain ischemia-reperfusion model was generated by the clipping of bilateral common carotid artery with hypotension. Dex $(9 \mu \mathrm{g} / \mathrm{kg})$ was infused immediately after reperfusion, while the other two groups received equal volume of saline. Morris water maze was employed to detect learning and memory function of rats. Brain tissues were extracted at 6, 24 and $72 \mathrm{~h}$ after reperfusion. Immunohistochemistry (IHC) was used to detect GluR2 and NR2B receptor levels, the mRNA levels of which were determined by real-time PCR.

Results: The longer escape latency and fewer number of crossing platform in water maze were shown in Model rats group. Cortical GluR2 expression was down-regulated while NR2B level was increased. Dex treatment inhibited escape latency and increased crossing times, along with the rise of cortical GluR2 expression and reduction of NR2B expression $(\mathbf{p}<0.05)$ in a time-dependent manner $(p<0.05)$.

Conclusion: Dex could alleviate ischemia-reperfusion injury of rat brain via down-regulating GluR2 while increasing NR2B expression.
\end{abstract}

Keywords: Dexmedetomidine, NMDA receptor 2B, Glutamate receptor 2, N-methyl-D-aspartic acid. Accepted on November 13, 2017

\section{Introduction}

With the aging of the population, cerebrovascular disease has emerged with an increasing trend of incidence, morbidity and mortality in recent years [1,2]. In clinics, cardiac- or neurosurgery has potential risk of cerebral ischemia. The most effective treatment is to regain blood perfusion, which, however, may cause secondary injury on brain tissues $[3,4]$. Brain ischemia-reperfusion damage is associated with a complicated patho-physiology process, which includes neurodegeneration, apoptosis or necrosis of neurons $[5,6]$. Neurotransmitter plays a critical role in the occurrence of ischemia cerebrovascular disease [7,8]. Some studies showed that during the process of brain ischemia, low neurotransmitter levels were found in hypothalamus, cerebral cortex and hippocampus, along with low neural activity, leading to cell apoptosis [9,10]. Hypoxia and insufficient perfusion caused intracellular $\mathrm{Ca}^{2+}$ imbalance. $\mathrm{Ca}^{2+}$ channel can be classified as Ligand-Gated Calcium Channel (LGCC) and Voltage-Gated Calcium Channel (VGCC). Excitatory neurotransmitter Glutamate (Glu) exerts its function mainly via binding on LGCC. Glutamate receptor includes both ion channel type and metabolic type, among which ion channel consists of $\mathrm{N}$ Methy-D-Aspartic Acid (NMDA) receptor, Kainic Acid (KA) receptor, and $\alpha$-amino-3-hydroxyl-5-methyl-4-isoxazolepropionic acid (AMPA) receptor. AMPA receptor participates in excitotoxicity of glutamate. It consists of four subunits (GluR1, GluR2, GluR3 and GluR4), which can alter the conductivity of channels. The percentage of GluR2 subunit can be increased during brain ischemia-reperfusion, thus significantly depress calcium permeability of AMPAR, leading to high ion influx and severe neuron damage [11-13]. NMDA receptor contains NR1, NR2, NR3 and NR4 subunits, among which NR2B contributes to a vital function in ischemiainduced neuronal damage $[14,15]$. Dexmedetomidine (Dex) is a type of selective $\alpha 2$-adernoglandin receptor agonist, and has been used as anesthetics or sedatives for critical patients. Accumulative evidence has shown that Dex could suppress inflammation via direct inhibition of inflammatory factors or activation cholinergic anti-inflammation pathway. However, its neuroprotective mechanism remains unclear yet $[16,17]$. This study aims to determine the effect as well as the mechanism of Dex on rat ischemia-reperfusion injury and related mechanism. 


\section{Materials and Methods}

\section{Animals and grouping}

Male healthy SD rats (7 w, body weight 220 250 g) were provided by laboratory animal center, Chinese Medicine Academy (Cert. No. SYXK-2013-0025) and were kept in an SPF-grade facility with food and water given ad libitum. Animals were randomly divided into sham, model and Dex group $(\mathrm{N}=20$ each). $9 \mu \mathrm{g} / \mathrm{kg}$ Dex was given intravenously immediately after re-perfusion. The other two groups received equal volumes of saline.

Rats were used for all experiments, and all procedures were approved by the Animal Ethics Committee of Third Xiangya Hospital of Central South University.

\section{Drugs and reagents}

Dex (200 $\mu \mathrm{g} / 2 \mathrm{ml}$, Hengrui Pharmaceutical, China); antiglutamate receptor (Chemicon Millipore, US); GluR2/NR2B rabbit $\mathrm{mAb}$ (Cell Signaling Technology, US); Secondary antibody (Boster, China); Primers for GluR2 and NR2B mRNA were synthesized by Invitrogen (US); DNA polymerase (Toyobo, China); Reverse transcription kit (Fermentas, US); Trizol reagent (Invitrogen, US); GAPDH antibody (Kangcheng Biotechnology, China). Morris water maze (DMS-2, Pharmaceutical Institute, Chinese Medicine Academy).

\section{Animal model}

Neural Defect Score (NDS) was examined to rule out those with congenital neural dysfunctions at $1 \mathrm{~h}$ before surgery. Cerebral ischemia-reperfusion model was generated as previously recorded [18]. In brief, the clipping of bilateral common carotid artery combined with hypotension was applied. Rats were fasted $8 \mathrm{~h}$ before surgery. After anesthesia by $10 \%$ chloral hydrate, rats were placed in supine position. Bilateral common carotid artery was exposed via mid-incision of the neck. Common carotid vein on right side and unilateral femoral artery were also separated with intact nerves. A cannula was placed via femoral artery puncture to observe blood pressure. A second cannula was inserted into carotid common artery toward the heart for blood collection and drug delivery. Venous blood was collected slowly via femoral common vein (rats had blood volume at 30 50\%) and average artery pressure was kept at $35 \sim 45 \mathrm{mmHg}$. Bilateral common carotid artery was closed by micro-clip. Electroencephalogram (EEG) was performed by insertion of electrodes on bilateral temporal skull. The successful generation of cerebral ischemia model was identified as the existence of static wave, pupil dilation or even the absence of cornea reflex. Bilateral common carotid artery was clipped for $15 \mathrm{~min}$, followed by backtransfusion of venous blood via common carotid vein at $1.2 \mathrm{ml} /$ min. In sham group, blood vessels were separated without ligation. Penicillin sodium was applied on the surgical site to prevent infection. Dex was diluted in saline and was infused into veins in a total volume of $9 \mu \mathrm{g} / \mathrm{kg}$ body weight. In the first hour, Dex was injected via common carotid vein at $3 \mu \mathrm{g} / \mathrm{kg}$, followed by $2 \mathrm{~h}$ infusion at $3 \mu \mathrm{g} / \mathrm{kg} / \mathrm{h}$. In the other two groups, equal volume of saline was applied.

\section{Behavioral test}

The neurobehavioral score of rats at $24 \mathrm{~h}$ after reperfusion was evaluated according to Bonilla's method [19] (mNss score), which includes sense, motion, balance and reflection, 0 , as the lowest score, represented no damage. The nerve injury became serious as score increased up to 18 . Water maze test includes both navigation session and space exploration session. The test apparatus is profiled with the water depth of $30 \mathrm{~cm}$ and temperature of $\left(20 \pm 2{ }^{\circ} \mathrm{C}\right)$. In navigation session, rats were trained for five consecutive days. After the training was completed for four days, the escape latency to climbing onto the platform was recorded. In exploration session, the swimming path and times of crossing the platform were recorded.

\section{NDS examination}

At $6 \mathrm{~h}, 24 \mathrm{~h}, 72 \mathrm{~h}$ after re-perfusion, NDS was performed, including vision, cognition, corena reflex, motor and seizure episode. A scale from 0 (normal) to 100 (brain death) was applied.

\section{Immunohistochemistry (IHC)}

Five rats were selected from all groups at each time point. After the rats were sacrificed, cerebellum and olfactory bulb from brains were collected. Cerebral cortex was separated on ice for preparing paraffin sections. After de-wax, three-step IHC staining (Ultra Vision Detection System) was performed to detect the expressions of GluR2 and NR2B using rabbit antirat GluR2 or NR2B receptor polyclonal antibody (1:500 dilution). After DAB staining, sections were counter-stained and mounted. Image-pro plus image analysis system was used to calculate the average optical density in six fields from each slide.

\section{RT-PCR}

Hippocampal tissues posterior of optic chiasm were extracted for preparing tissue homogenate. TransZol Up method was applied to quantify total RNA. Using cDNA as the template, PCR amplification was performed under the following conditions: $94^{\circ} \mathrm{C}$ per-denature for $5 \mathrm{~min}$, followed by 30 cycles each containing $94^{\circ} \mathrm{C}$ denature for $30 \mathrm{~s}, 57^{\circ} \mathrm{C}$ annealing for 30 $\mathrm{s}$ and $72^{\circ} \mathrm{C}$ elongation for $20 \mathrm{~s}$. Using GAPDH as the internal reference, semi-quantitative analysis was performed by gel imaging analysis system to calculate relative absorption value. Primer sequences were shown in Table 1.

\section{Statistical method}

SPSS 20.0 software was applied for statistical analysis. Measurement data were firstly tested for normality. Those fitted normal distribution were presented as mean \pm Standard Deviation (SD). Analysis of Variance (ANOVA) was used to compare means across multiple groups. LSD test was used for 
The effect of dexmedetomidine on GluR2 and NR2B expression of cortical neurons after cerebral ischemia-reperfusion in rats

between-group-comparison. A statistical significance was defined when $\mathrm{p}<0.05$.

\section{Results}

\section{Cerebral ischemia-perfusion impaired rat learning and memory function}

According to the data of neurobehavioral scoring, the nerve injury was severer in model group $(9.46 \pm 0.55)$ than that in the sham group $(2.41 \pm 0.45)$. However, Dex alleviated the nerve damage caused by cerebral ischemia-perfusion $(7.31 \pm 0.63)$ $(\mathrm{P}<0.05)$. Compared to sham group, significantly high escape latency and few times of platform crossing in model rats were shown $(\mathrm{p}<0.05)$. However, Dex treatment statistically shortened escape latency and increased times of platform crossing ( $\mathrm{p}<0.05$ compared to model group, Figure 1).
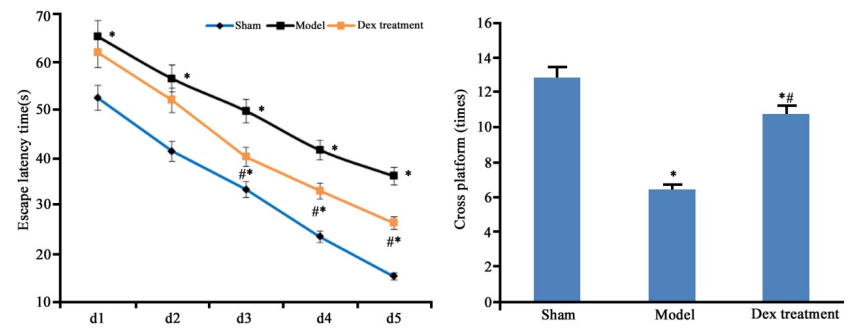

Figure 1. Water-maze performance of rats. Left panel, escape latency; Right panel, number of crossing platform. ${ }^{*} p<0.05$ compared to control group; ${ }^{\#} p<0.05$ compared to model group.

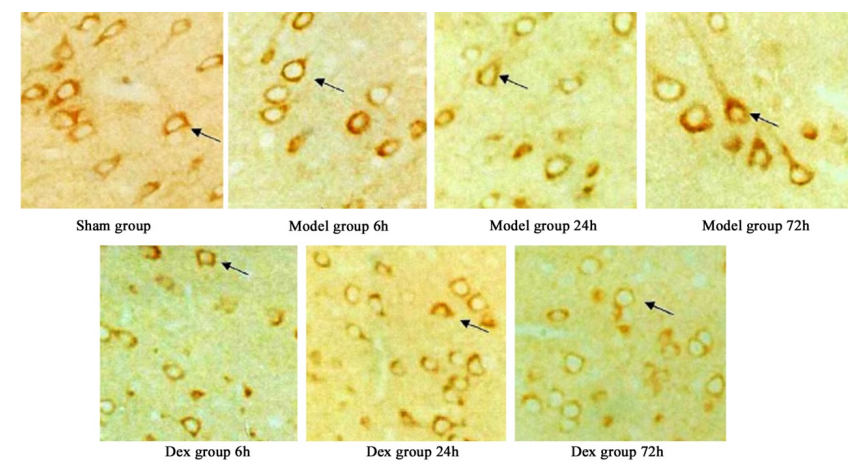

Figure 2. The expression of GluR2 receptor in cerebral cortex (IHC staining, X400).

\section{The expressions of GluR2 and NR2B receptor in cerebral cortex}

In model group, the expression of GluR2 receptor was significantly reduced the level of NR2B receptor was statistically elevated compared to that in sham group $(\mathrm{p}<0.05)$. No significant difference was found regarding GluR2 receptor level at different time points. Of note, in Dex treatment group, the level of GluR2 receptor along with the decrease of NR2B receptor expression in cortical neurons was observed compared to that in model group and significant difference of expressions was exhibited at different time points ( $p<0.05$, Figures 2-4).

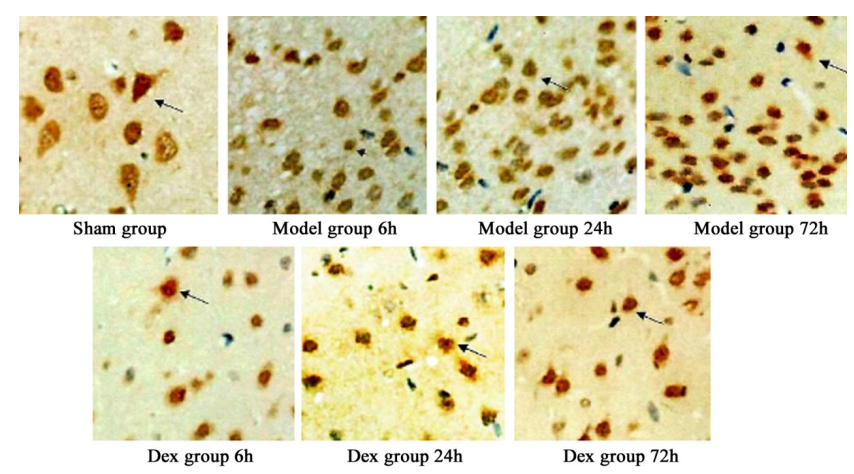

Figure 3. The expression of NR2B receptor in cerebral cortex (IHC staining, X400).
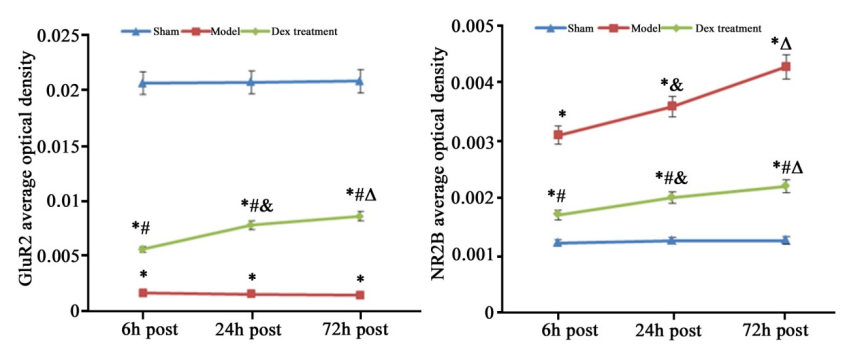

Figure 4. The expressions of GluR2 (upper panel) and NR2B (lower panel) receptors. ${ }^{*} p<0.05$ compared to control group; ${ }^{*} p<0.05$ compared to model group; ${ }^{\star} p<0.05$ compared to 6 h group; ${ }^{\Delta} p<0.05$ compared to 24 h group.
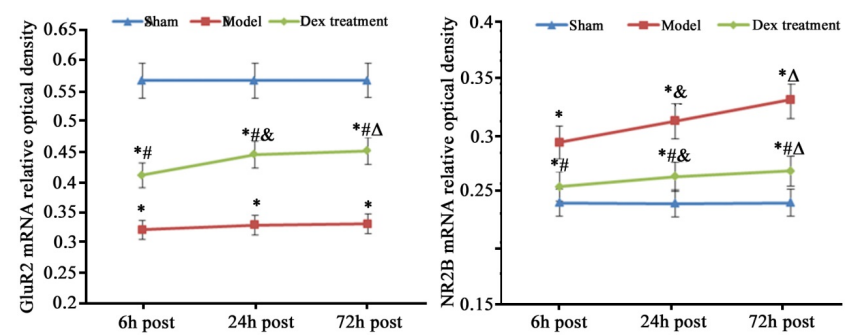

Figure 5. The expressions of GluR2 (upper panel) and NR2B (lower panel) mRNAs. ${ }^{*} p<0.05$ compared to control group; ${ }^{*} p<0.05$ compared to model group; ${ }^{\&} p<0.05$ compared to 6 h group; ${ }^{\Delta} p<0.05$ compared to 24 hroup.

\section{mRNA expression level of GIuR2 and NR2B in cerebral cortex}

As consistent with data of IHC staining, compared to sham group, cerebral ischemia-reperfusion in model group induced the statistically decrease of GluR2 and rise of NR2B mRNA expression $(\mathrm{p}<0.05)$. The treatment of Dex significantly elevated GluR2 mRNA and inhibited NR2B mRNA expression in cortical neurons damaged by ischemia-reperfusion compared 
to that in the model group without the treatment of Dex $(\mathrm{p}<0.05$, Figure 5).

Table 1. Primer sequence.

\begin{tabular}{llll}
\hline Gene & Length & Forward primer & Reverse primer \\
\hline NR2B & $227 \mathrm{bp}$ & 5'-AGCTGCTCTCCACACCCTGAGAT-3' & 5'-ACGCCAAACCTCTAGGCGGACA-3' \\
\hline GluR2 & $93 \mathrm{bp}$ & 5'-AGCAGATTTAGCCCCTACGAG-3' & 5'-TAAGTTAGCCGTGTAGGAGGA-3' \\
\hline GAPDH & $317 \mathrm{bp}$ & 5'-ATCACTGCCACTCAGAAG-3' & 5'-AAGTCACAGGAGACAACC-3' \\
\hline
\end{tabular}

\section{Discussion}

Ischemia-reperfusion injury of brain is a type of common pathological change in clinics and severely affects patient's life quality. The alleviation of brain ischemia-reperfusion damage is thus of great importance for the therapy. Dex exerts to multiple functions including sedative, anti-inflammation, and neuroprotection, although its neuroprotective mechanism has not been illustrated fully [17]. During the process of wholebrain ischemia-reperfusion injury, hippocampal CA1 and CA3 regions are most sensitive for ischemia, in which primary neuron apoptosis usually occurred at $3 \mathrm{~h}$ after re-perfusion. The rate of cell apoptosis reaches the peak between 24 and $48 \mathrm{~h}$, while cell necrosis increases at $48 \mathrm{~h}$ and the peak level was apparent at $72 \mathrm{~h}$ [19]. This study thus selected three different time points: 6,24 and $72 \mathrm{~h}$ after re-perfusion, and investigated the neuroprotective function of Dex. AMPA receptor is composed of GluR1, GluR2, GluR3 and GluR4 subunits, which are encoded by different genes. The relative content of GluR2 subunit affects AMPA receptor function through the mediation of the permeability of Calcium ion. In most neurons, GluR2 subunit is highly expressed, and contributes to the inhibition of the calcium ion influx via AMPA receptor. Neural injury decreases GluR2 expression and thus elevates Calcium ion influx. After ischemia or excitotoxicity, GluR2 expression is decreased in survival neurons [20]. NR1 represents a basic functional unit of NMDA receptor, while NR2 is of great importance for regulatory function of NMDA receptor, which is correlated with neuronal excitotoxicity, neuronal injury after brain ischemia-reperfusion [21].

AMPA receptor mediates the permeability of calcium ion in neurons. Cerebral ischemia in rats increases the level of AMPA receptor without GluR2 subunit in hippocampal neurons. The decreasing amount of AMPA receptor containing GluR2 subunit leads to calcium influx and neuronal apoptosis. After NMDA receptor is activated, the calcium homeostasis across cell membrane is disrupted, resulting in neuronal apoptosis. This study showed reducing expression of GluR2 in cortical neurons in model group at all-time points. Dex treatment nevertheless elevated GluR2 expression in neurons, suggesting the significant role of GluR2 in cell injury and calcium overload induced by cerebral ischemia-reperfusion. The declining expression of GluR2 initiates calcium influx and causes cellular calcium overload. Multiple mechanisms may induce calcium overload in neurons after ischemia. Dex on the other hand may exert neuroprotective role via inhibiting the elevation of NR2B expression in cortical neurons during cerebral ischemia-reperfusion of rats. Our RT-PCR results showed consistent changes of mRNA levels of GluR2 and NR2B in comparison with the data of IHC staining. GluR2 and NR2B affect calcium ion during neuronal injury caused by ischemia-reperfusion, and synergistically induce cellular calcium ion influx. NMDA receptor also participates in ischemia brain injury with L-type calcium ion channel.

In summary, Dex could alleviate cerebral ischemia-reperfusion injury in rats, possibly via inhibiting the down-regulation of GluR2 and over-expression of NR2B during the process of ischemia-reperfusion damage.

\section{References}

1. Lantz M, Sjostrand C, Kostulas K. Ischemic stroke and patent foramen ovale: risk factors and genetic profile. J Stroke Cerebrovasc Dis 2013; 22: 841-845.

2. Baldi I, Costa G, Foltran F, Spada V, Chiffi D, Sacerdote C, Gregori D. Effect of educational attainment on incidence and mortality for ischemic heart and cerebrovascular diseases: a systematic review and trend estimation. Int $\mathrm{J}$ Cardiol 2013; 168: 4959-4963.

3. OBrien JT, Thomas A. Vascular dementia. Lancet 2015; 386: 1698-1706.

4. Ueno M, Chiba Y, Matsumoto K, Murakami R, Fujihara R, Kawauchi M, Miyanaka H, Nakagawa T. Blood-brain barrier damage in vascular dementia. Neuropathology 2016; 36: 115-124.

5. Makarewicz D, Slomka M, Danysz W, Lazarewicz JW. Effects of mGluR5 positive and negative allosteric modulators on brain damage evoked by hypoxia-ischemia in neonatal rats. Folia Neuropathol 2015; 53: 301-308.

6. Lv Y, Qian Y, Ou-Yang A, Fu L. Hydroxysafflor yellow a attenuates neuron damage by suppressing the lipopolysaccharide-induced tlr4 pathway in activated microglial cells. Cell Mol Neurobiol 2016; 36: 1241-1256.

7. Zanelli SA, Rajasekaran K, Grosenbaugh DK, Kapur J. Increased excitability and excitatory synaptic transmission during in vitro ischemia in the neonatal mouse hippocampus. Neuroscience 2015; 310: 279-289.

8. Zhitkova JV. Comparison of different doses of escitalopram in the prevention of dementia in patients with depression and moderate cognitive dysfunction associated with chronic 
brain ischemia. Zh Nevrol Psikhiatr Im S S Korsakova 2015; 115: 53-60.

9. Sun Y, Zhang L, Chen Y, Zhan L, Gao Z. Therapeutic targets for cerebral ischemia based on the signaling pathways of the GluN2B C terminus. Stroke 2015; 46: 2347-2353.

10. Mirzoyan NR, Hostikyan NG, Stepanyan AG, Akopyan AA, Alikhanyan KB, Meliksetyan VS, Bagdasaryan NA, Gretskaya NM, Bezuglov VV. Studying neuroprotective activity of GABA conjugate with arachidonic acid under conditions of local permanent brain ischemia in rats. Eksp Klin Farmakol 2015; 78: 7-11.

11. Chen X, Li H, Huang M, Huang M, Xu W, Chu K, Chen L, Zhang Y. Effect of Gua Lou Gui Zhi decoction on focal cerebral ischemia-reperfusion injury through regulating the expression of excitatory amino acids and their receptors. Mol Med Rep 2014; 10: 248-254.

12. Noh KM, Yokota H, Mashiko T, Castillo PE, Zukin RS, Bennett MV. Blockade of calcium-permeable AMPA receptors protects hippocampal neurons against global ischemia-induced death. Proc Natl Acad Sci USA 2005; 102: 12230-12235.

13. Wang XQ, Zhong XL, Li ZB, Wang HT, Zhang J, Li F, Zhang JY, Dai RP, Xin-Fu Z, Li CQ, Li ZY, Bi FF. Differential roles of hippocampal glutamatergic receptors in neuropathic anxiety-like behavior after partial sciatic nerve ligation in rats. BMC Neurosci 2015; 16: 14.

14. Pages N, Maurois P, Delplanque B, Bac P, Vamecq J. Brain anticonvulsant protection of mice given chronic carbamazepine under various fatty acid and magnesium diet conditions. Prostaglandins Leukot Essent Fatty Acids 2012; 87: 63-70.

15. Pages N, Maurois P, Delplanque B, Bac P, Martin JC, Du Q, Rapoport SI, Vamecq J. Brain protection by rapeseed oil in magnesium-deficient mice. Prostaglandins Leukot Essent Fatty Acids 2011; 85: 53-60.

16. Chi OZ, Grayson J, Barsoum S, Liu X, Dinani A, Weiss HR. Effects of dexmedetomidine on microregional $\mathrm{O} 2$ balance during reperfusion after focal cerebral ischemia. J Stroke Cerebrovasc Dis 2015; 24: 163-170.

17. Wang Z, Kou D, Li Z, He Y, Yu W, Du H. Effects of propofol-dexmedetomidine combination on ischemia reperfusion-induced cerebral injury. Neuro Rehab 2014; 35 : 825-834.

18. Brambrink AM, Koerner IP, Diehl K, Strobel G, Noppens $\mathrm{R}$, Kempski $\mathrm{O}$. The antibiotic erythromycin induces tolerance against transient global cerebral ischemia in rats (pharmacologic preconditioning). Anesthesiology 2006; 104: 1208-1215.

19. Giacoppo S, Galuppo M, Iori R, De Nicola GR, Bramanti P, Mazzon E. (RS)-glucoraphanin purified from Tuscan black kale and bioactivated with myrosinase enzyme protects against cerebral ischemia/reperfusion injury in rats. Fitoterapia 2014; 99: 166-177.

20. Harlow DE, Saul KE, Komuro H, Macklin WB. Myelin proteolipid protein complexes with alphav integrin and AMPA receptors in vivo and regulates ampa-dependent oligodendrocyte progenitor cell migration through the modulation of cell-surface GluR2 expression. J Neurosci 2015; 35: 12018-12032.

21. Wang H, Liu S, Wang H, Wang G, Zhu A. The effect of propofol postconditioning on the expression of $\mathrm{K}(+)-\mathrm{Cl}(-)-$ co-transporter 2 in GABAergic inhibitory interneurons of acute ischemia/reperfusion injury rats. Brain Res 2015; 1597: 210-219.

\section{*Correspondence to}

Yanwen $\mathrm{Li}$

Department of Anesthesiology

Third Xiangya Hospital of Central South University

PR China 